\title{
The Complement Receptor C5aR Has a Dual, Time- Dependent Effect on the Outcome of Spinal Cord Injury
}

\author{
(ㄱ)Antigona Ulndreaj, ${ }^{1,3}$ - Jessica M. Marbourg, ${ }^{2}$ and Pia M. Vidal ${ }^{3}$ \\ ${ }^{1}$ Institute of Medical Science, Faculty of Medicine, University of Toronto, Toronto, Ontario M5T 2S8, Canada, ${ }^{2}$ Neuroscience Graduate Program and Center \\ for Brain and Spinal Cord Repair, The Ohio State University, Columbus, Ohio 43210, and ${ }^{3}$ Division of Genetics and Development, Toronto Western \\ Research Institute and Spinal Program, Krembil Neuroscience Centre, University Health Network, Toronto, Ontario M5T 2S8, Canada \\ Review of Brennan et al.
}

The complement system consists of more than 30 plasma and membrane-bound proteins that promote host defense by inducing cell lysis, disposing of immune complexes, and augmenting the adaptive immune response. Under homeostatic conditions, these complement proteins are expressed by all the cells of the CNS at low levels. However, upon insult (infection, injury, or ischemia) complement proteins are upregulated, and if the balance between complement activation and regulation is disturbed, the CNS parenchyma can be subjected to detrimental complement-mediated attack. The complement cascade is a series of enzymatic reactions that can be activated through at least three different pathways - the classical, the alternative, and the lectin pathway-all of which involve the generation of C5a. Specifically, in one step of the complement cascade, C5 protein is fragmented into C5a and C5b. While C5b participates in lysis of pathogenic microbes by forming the membrane attack complex (MAC) on their cell surface, C5a acts as a potent chemoattractant of inflammatory cells at the site of its generation (Peterson and Anderson, 2014). C5a is upregulated immediately after CNS injury in ro-

Received June 22, 2015; revised Aug. 3, 2015; accepted Aug. 4, 2015.

We thank Drs. Michael G. Fehlings and Phillip G. Popovich for providing critical feedback during the preparation of this manuscript and Dr. Madeleine $0^{\prime}$ Higgins for assistance with editing.

Correspondence should be addressed to Antigona Ulndreaj: Krembil Discovery Tower, 60 Leonard Avenue, 7KD430, Toronto, ON M5T 258, Canada.E-mail: ulantig@gmail.com.

DOI:10.1523/JNEUROSCI.2380-15.2015

Copyright $\odot 2015$ the authors $\quad 0270-6474 / 15 / 3512325-03 \$ 15.00 / 0$ dents and humans (Peterson and Anderson, 2014), but its role in CNS pathologies remains unclear. Some data suggest a protective effect of C5a signaling (Weerth et al., 2003; Beck et al., 2010; Guo et al., 2013) while other data suggest the opposite (Li et al., 2014). These divergent effects of C5a signaling might also be time-dependent (Beck et al., 2010; Guo et al., 2013).

A recent study by Brennan et al. (2015) provides compelling in vivo evidence in a mouse model of T9 spinal cord injury (SCI) that resolves much of the uncertainty surrounding the effect and time dependency of C5a signaling after CNS trauma. Specifically, during the acute injury phase [before $7 \mathrm{~d}$ post injury (dpi)], pharmacological blockade or genetic ablation of the $\mathrm{C} 5 \mathrm{a}$ receptor (C5aR) significantly improved functional recovery. These functional benefits corresponded with reduced inflammatory cytokine levels and monocyte-derived macrophage infiltration early after injury. Conversely, prolonged (C5aR blockade up to $21 \mathrm{dpi}$ ) or permanent ( $C 5$ ar knock-out) disruption of $\mathrm{C} 5 \mathrm{aR}$ signaling impaired formation of the astrogliotic scar around the lesion core, exacerbated peripheral immune cell infiltration and lesion size, and reduced functional recovery. These effects were shown to be mediated in part by C5aR-dependent activation of STAT3, a transcription factor necessary for promoting astrocyte proliferation. Collectively, these data indicate that $\mathrm{C} 5 \mathrm{aR}$ signaling is detrimental during acute injury, but is necessary for protective glial scar formation chronically (Fig. 1).

$\mathrm{C} 5 \mathrm{aR}$ antagonism restricted to the first 7 dpi improved functional outcomes, increased myelination, and reduced immune cell infiltration at $35 \mathrm{dpi}$ (Brennan et al., 2015, their Fig. 3). In this condition, inhibiting C5aR signaling did not affect astrogliosis, but it reduced immediate postinjury increases of inflammatory cytokines, which are thought to be integral for the recruitment of immune cells. To confirm this, and in an attempt to restrict the effects of $\mathrm{C} 5 \mathrm{aR}$ signaling disruption to cells in the CNS, Brennan et al. (2015) used bone marrow chimeric mice in which C5ar-deficient bone marrow cells were transplanted into irradiated wildtype mice. In these animals, C5aR signaling deficiency was restricted to cells of the peripheral immune system. Importantly, myelin content and functional recovery after SCI in these mice was not different from control irradiated wild-type mice that received wild-type bone marrow transplants (Brennan et al., 2015, their Fig. 5). This suggests that, to achieve neuroprotection, C5aR disruption must target CNS-resident cells.

The observation that eliminating peripheral $\mathrm{C} 5 \mathrm{aR}$ signaling does not affect SCI outcomes is critical given that infiltrating immune cells have been directly associated with changes in pathology and functional recovery (Popovich et al., 


\section{Outcome at 35 days post $\mathrm{SCl}$}

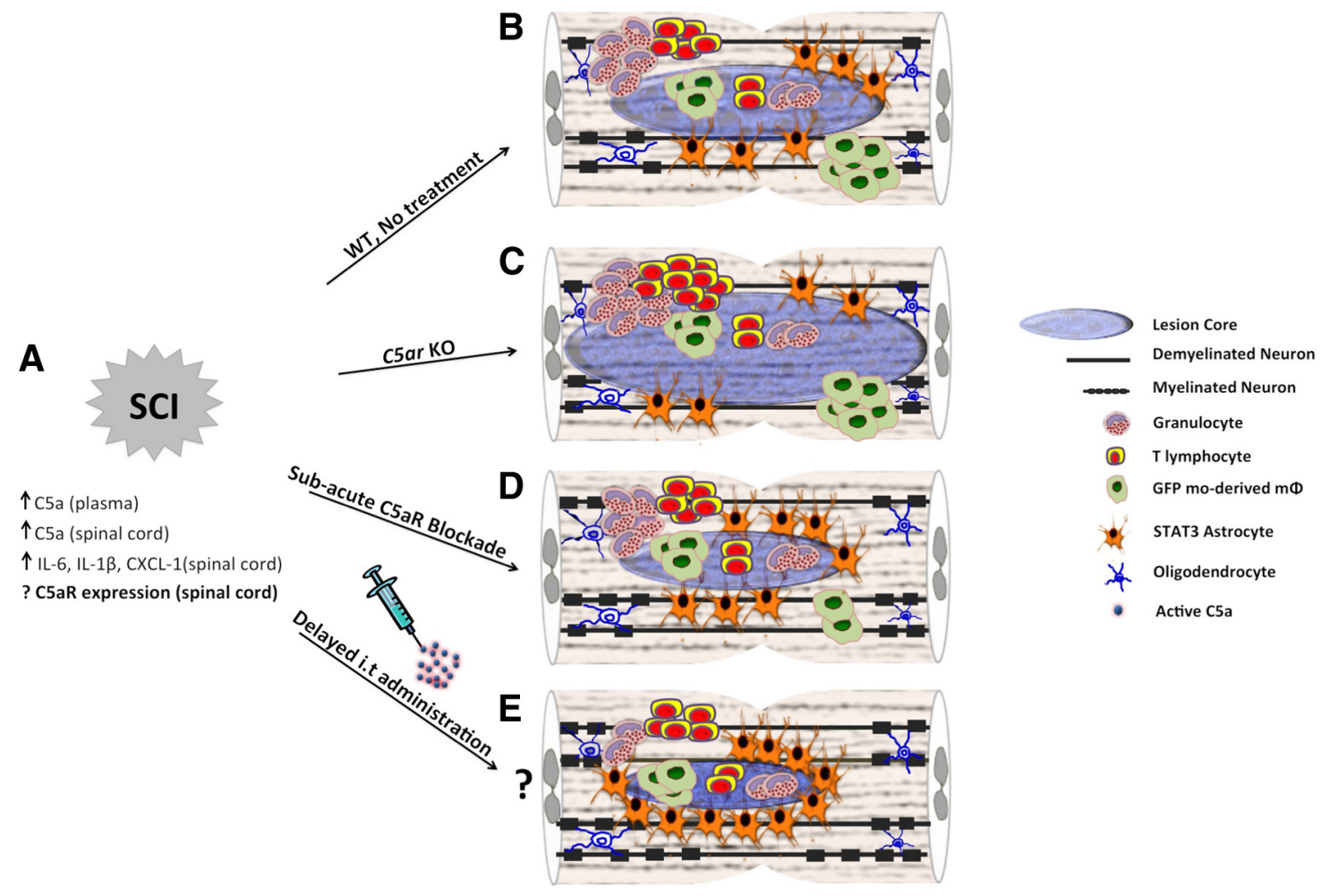

Figure 1. Schematic diagram showing diverse time-dependent therapeutic effects of $\mathrm{C} 5$ a signaling after SCI. A, Immediately after SCI, C5a levels in plasma and the spinal cord increase, resulting in the subsequent upregulation of intraspinal inflammatory mediators (IL-6, IL-1 $\beta$, and CXCL1). They, in turn, recruit peripheral immune cells to the injury site. The effects of C5a are relayed through C5aR, which is also expressed in the CNS, although its expression kinetics have not been described. B, In wild-type (WT) mice, SCl results in the formation of a lesion core containing infiltrating immune cells. Astrocytes are also recruited to the lesion core to form the glial scar. C, In C5ar knock-out (KO) mice or upon long-term $(5 a R$ pharmacological blockade, there are significant increases in lesion size, demyelination, and recruitment of T lymphocytes and granulocytes. Lack of $C 5$ aR signaling reduces astrocyte proliferation and glial scar formation through STAT3 signaling. D, Blockade of $\mathrm{C} 5 \mathrm{aR}$ during the subacute injury phase presents an effective therapeutic time window to reduce recruitment of monocyte-derived macrophages (mo-derived $\mathrm{m} \Phi)$, demyelination, and lesion size. $\boldsymbol{E}$, Given the dichotomous effect of $\mathrm{C}$ aR in $\mathrm{SCl}$, it would be useful to explore whether delayed intrathecal (i.t) administration of C5a could promote recovery by increasing proliferation of STAT3-dependent astrocytes, which in turn, will prevent the spread of secondary injury.

1999). Although Brennan et al. (2015) show that ubiquitous C5aR deficiency ameliorates postinjury induction of inflammatory cytokines (Brennan et al., 2015, their Fig. 4A-F), and that systemic pharmacological blockade of $\mathrm{C} 5 \mathrm{aR}$ significantly reduces peripheral immune cell recruitment to the injured spinal cord at 35 dpi (Brennan et al., 2015, their Fig. 3 E, G), their bone-marrow chimera results suggest that these effects are CNS-dependent. From these data, one can conclude that acute C5aR signaling in CNS cells contributes to the onset of intraspinal inflammation after SCI. Given the role of CNS cells in initiating C5aR signaling after SCI, it would have been useful for the authors to quantify the temporal C5aR expression in the spinal cord postinjury. Although they include histology of $\mathrm{C} 5 \mathrm{aR}$ expression on microglia/macrophages and astrocytes at 1 and 7 dpi (Brennan et al., 2015, their Fig. 1), similar data at later time points are not provided. This would have been particularly useful after the subacute injury phase when $\mathrm{C} 5 \mathrm{aR}$ signaling is proposed to be important for astrocyte proliferation and eventual recovery of function.

The study by Brennan et al. (2015) provides interesting insights that could fuel future research on three main fronts, with time after SCI as the common theme.

First, astrogliosis is not always harmful. Although it has often been viewed as detrimental to the outcome of SCI due to its inhibitory effect on axonal regrowth (Menet et al., 2003), recent studies have challenged this notion, emphasizing that astrocytes are heterogeneous and some astrocyte activation is neuroprotective (Faulkner et al., 2004). For example, in a recent SCI study, astrocytes that were located in the scar border surrounding the lesion core were described as elongated, proliferating cells, whereas the hypertrophic astrocytes showing less proliferation were found $>1 \mathrm{~mm}$ away from the lesion core (Wanner et al., 2013). In their study, Brennan et al. (2015) allowed for protective C5aR-mediated astrocyte proliferation without causing excessive astrogliosis by limiting $\mathrm{C} 5 \mathrm{aR}$ inhibition to the sub- acute injury phase. Importantly, in vitro data show that C5aR is involved in inducing proliferation of STAT3-expressing astrocytes, which have been shown to limit the spread of inflammation and neuronal loss in the injured spinal cord by contributing to scar formation (Okada et al., 2006; Herrmann et al., 2008; Wanner et al., 2013). This suggests that C5aR signaling might favor proliferation of protective astrocytes after SCI. Together, the study highlights the importance of harnessing the beneficial effects of astrogliosis for developing successful therapies for SCI.

Second, C5aR signaling might promote regeneration after SCI. One of the obstacles to overcome in the treatment of CNS injury is resistance of the mammalian CNS to regenerate. Interestingly, C5a has been implicated as an instrumental mediator of hepatocyte proliferation and liver regeneration via activation of the STAT3 pathway (Strey et al., 2003). It is therefore conceivable that $\mathrm{C} 5 \mathrm{aR}$ signaling could have a regenerative role after CNS injury. Indeed, a study by Guo et al. 
(2013) reported that C5a enhanced neuronal outgrowth in vitro and improved functional recovery upon its delayed administration after SCI in mice. In combination with this literature, the findings by Brennan et al. (2015) suggesting a dual, time-dependent role of C5aR create optimism that exogenous stimulation of $\mathrm{C} 5 \mathrm{aR}$ may further enhance recovery in SCI by direct promotion of regeneration (Fig. 1E).

Third, SCI outcomes appear to be primarily influenced by central C5aR signaling, and its therapeutic manipulation can therefore be restricted to the CNS and so should its therapeutic manipulation. Brennan et al. (2015) present the novel finding that inhibiting $\mathrm{C} 5 \mathrm{aR}$ signaling can be neuroprotective only when these effects are confined to the CNS. Thus, future therapeutic approaches could focus on strategies that manipulate $\mathrm{C} 5 \mathrm{aR}$ signaling exclusively in the CNS, to avoid off-target effects in the periphery. Moreover, the notion among spine surgeons that "time is spine" regarding the management of human SCI is also reflected in the work by Brennan et al. (2015), where immediate and transient blockade of C5aR signaling resulted in persisting improvement (Fig. 1D). Most importantly, by emphasizing the dichotomous role of $\mathrm{C} 5 \mathrm{aR}$ in the progression of SCI, the results of Brennan et al. (2015) should prompt future studies to investigate whether exogenous $\mathrm{C} 5 \mathrm{aR}$ stimulation in the chronic phase will improve outcomes after SCI (Guo et al., 2013). This approach could provide a dual therapeutic strategy to enhance recovery in SCI patients and address the long-standing need for treatment of chronic SCI (Fig. 1E).
In summary, the pivotal role of the immune system in the progression of SCI is well established. However, SCI-induced immune activation is not an all or none response that kills or repairs injured tissue. Instead, it is an intricate and complex process that requires precise tuning to harness its beneficial effects. Brennan et al. (2015) reveal that C5aR has an important CNS-restricted and time-dependent role in recovery after experimental SCI, and these findings should encourage future research to avoid traditional blackor-white approaches in the treatment of SCI.

\section{References}

Beck KD, Nguyen HX, Galvan MD, Salazar DL, Woodruff TM, Anderson AJ (2010) Quantitative analysis of cellular inflammation after traumatic spinal cord injury: evidence for a multiphasic inflammatory response in the acute to chronic environment. Brain 133:433447. CrossRef Medline

Brennan FH, Gordon R, Lao HW, Biggins PJ, Taylor SM, Franklin RJ, Woodruff TM, Ruitenberg MJ (2015) The complement receptor C5aR controls acute inflammation and astrogliosis following spinal cord injury. J Neurosci 35:6517-6531. CrossRef Medline

Faulkner JR, Herrmann JE, Woo MJ, Tansey KE, Doan NB, Sofroniew MV (2004) Reactive astrocytes protect tissue and preserve function after spinal cord injury. J Neurosci 24:21432155. CrossRef Medline

Guo Q, Cheng J, Zhang J, Su B, Bian C, Lin S, Zhong C (2013) Delayed post-injury administration of $\mathrm{C} 5 \mathrm{a}$ improves regeneration and functional recovery after spinal cord injury in mice. Clin Exp Immunol 174:318325. Medline

Herrmann JE, Imura T, Song B, Qi J, Ao Y, Nguyen TK, Korsak RA, Takeda K, Akira S, Sofroniew MV (2008) STAT3 is a critical regulator of astrogliosis and scar formation after spinal cord injury. J Neurosci 28:72317243. CrossRef Medline
Li L, Xiong ZY, Qian ZM, Zhao TZ, Feng H, Hu S, Hu R, Ke Y, Lin J (2014) Complement C5a is detrimental to histological and functional locomotor recovery after spinal cord injury in mice. Neurobiol Dis 66:74-82. CrossRef Medline

Menet V, Prieto M, Privat A, Giménez y Ribotta M (2003) Axonal plasticity and functional recovery after spinal cord injury in mice deficient in both glial fibrillary acidic protein and vimentin genes. Proc Natl Acad Sci U S A 100: 8999-9004. CrossRef Medline

Okada S, Nakamura M, Katoh H, Miyao T, Shimazaki T, Ishii K, Yamane J, Yoshimura A, Iwamoto Y, Toyama Y, Okano H (2006) Conditional ablation of Stat3 or Socs 3 discloses a dual role for reactive astrocytes after spinal cord injury. Nat Med 12:829-834. CrossRef Medline

Peterson SL, Anderson AJ (2014) Complement and spinal cord injury: traditional and nontraditional aspects of complement cascade function in the injured spinal cord microenvironment. Exp Neurol 258:35-47. CrossRef Medline

Popovich PG, Guan Z, Wei P, Huitinga I, van Rooijen N, Stokes BT (1999) Depletion of hematogenous macrophages promotes partial hindlimb recovery and neuroanatomical repair after experimental spinal cord injury. Exp Neurol 158:351-365. CrossRef Medline

Strey CW, Markiewski M, Mastellos D, Tudoran R, Spruce LA, Greenbaum LE, Lambris JD (2003) The proinflammatory mediators C3a and C5a are essential for liver regeneration. J Exp Med 198:913-923. CrossRef Medline

Wanner IB, Anderson MA, Song B, Levine J, Fernandez A, Gray-Thompson Z, Ao Y, Sofroniew MV (2013) Glial scar borders are formed by newly proliferated, elongated astrocytes that interact to corral inflammatory and fibrotic cells via STAT3-dependent mechanisms after spinal cord injury. J Neurosci 33: 12870-12886. CrossRef Medline

Weerth SH, Rus H, Shin ML, Raine CS (2003) Complement C5 in experimental autoimmune encephalomyelitis (EAE) facilitates remyelination and prevents gliosis. Am J Pathol 163:1069-1080. CrossRef Medline 